NONCOMMUTATIVE GEOMETRY

AND QUANTUM GROUPS

BANACH CENTER PUBLICATIONS, VOLUME 61

INSTITUTE OF MATHEMATICS

POLISH ACADEMY OF SCIENCES

WARSZAWA 2003

\title{
WEAK HOPF ALGEBRAS AND QUANTUM GROUPOIDS
}

\author{
PETER SCHAUENBURG \\ Mathematisches Institut der Universität München \\ Theresienstr. 39, 80333 München, Germany \\ E-mail: schauen@mathematik.uni-muenchen.de
}

\begin{abstract}
We give a detailed comparison between the notion of a weak Hopf algebra (also called a quantum groupoid by Nikshych and Vainerman), and that of a $\times_{R}$-bialgebra due to Takeuchi (and also called a bialgebroid or quantum (semi)groupoid by $\mathrm{Lu}$ and $\mathrm{Xu}$ ). A weak bialgebra is the same thing as a $\times_{R}$-bialgebra in which $R$ is separable. We extend the comparison to cover module and comodule theory, duality, and the question when a bialgebroid should be called a Hopf algebroid.
\end{abstract}

1. Introduction. Quantum groupoids (or Hopf algebroids) are to groupoids what quantum groups (or Hopf algebras) are to groups: A Hopf algebroid is the noncommutative analog of the function algebra on a groupoid.

A groupoid is a small category, and has a set of morphisms and a set of objects (in other terminology arrows and vertices). Thus the definition of a quantum groupoid should involve two algebras, one of which (say $H$ ) plays the role of the function algebra on the quantum space of morphisms, and the other (say $R$ ) the role of the function algebra on the quantum space of objects. Since there is a source and target assigned to each arrow, one should also expect (in the reverse direction) two maps from $R$ to $H$ to be part of the structure, while composition in the groupoid, a partially defined map on the product, should correspond to a comultiplication into a suitably defined tensor product of $H$ with itself.

In this note we will compare in detail two notions of quantum (semi)groupoids: the $\times_{R}$-bialgebras defined by Takeuchi [20], and the weak bialgebras defined by Böhm and Szlachányi [2]. Thus we shall, as it were, provide reference [NS] in [6]; while my joint paper with Florian Nill is announced there optimistically as a preprint to appear shortly, in reality it was never finished. The main result is as follows: A weak bialgebra is the same thing as a $\times_{R}$-bialgebra in which the algebra $R$ is separable.

The $\times_{R}$-bialgebras defined by Takeuchi [20], following work of Sweedler [19], are the first quantum (semi)groupoids appearing in the literature. One should note, though, that

2000 Mathematics Subject Classification: Primary 16W30.

The paper is in final form and no version of it will be published elsewhere. 
Takeuchi did not consider the analogy with groupoids at all, whereas this was the key motivation for the definitions of $\mathrm{Lu}[10]$ and $\mathrm{Xu}$ [21], which turn out to be equivalent to Takeuchi's, mostly by a translation of notations, though some care has to be taken about the somewhat different definitions of counits. See the paper of Brzeziński and Militaru [4] for details.

Weak Hopf algebras were defined by Böhm and Szlachányi [2], see also the recent survey [14] by Nikshych and Vainerman and the literature cited there. A weak bialgebra $H$ is a coalgebra and algebra such that the comultiplication is multiplicative, but does not preserve the unit; dually the multiplication is not counital. These two requirements are replaced by certain weakened versions. In this definition there is in the beginning no auxiliary algebra $R$ playing the role of the function algebra on the set of vertices, but rather two anti-isomorphic "source and target counital subalgebras" are constructed from the axioms.

A special case of weak bialgebras, called face algebras, had been defined earlier by Hayashi $[7,8]$. A face algebra turns out to be precisely the special case of a weak bialgebra in which the, say target, counital subalgebra is commutative. In [17] we have shown that a face algebra is precisely the special case of a $\times_{R}$-bialgebra in which the algebra $R$ is commutative and separable.

It turns out that one can show by essentially the same calculations that a weak bialgebra is precisely the special case of a $\times_{R}$-bialgebra in which $R$ is separable. A major difference to our previous considerations in [17] is that Hayashi's face algebras involve a commutative separable base algebra by definition, while one has to show that the target counital subalgebra of a weak bialgebra is separable. More precisely, it comes equipped with a specific separability idempotent which is at the same time part of a Frobenius system - we shall call this data an idempotent Frobenius system. For the other direction, that is, the construction of a weak bialgebra from a $\times_{R}$-bialgebra with separable $R$, we have to invoke a result of Kadison and Szlachányi, which says that an idempotent Frobenius system always exists. Contrary to the case of commutative $R$, not every separability idempotent is suitable, however.

The fact that any weak Hopf algebra is a $\times_{R}$-bialgebra (in fact a Hopf algebroid in the sense of $\mathrm{Lu}$ ) has meanwhile been shown by Etingof and Nikshych [5, Prop. 2.3.1], who also show that the target counital subalgebra is separable (note however that the formulas between (10) and (11) there seem to claim that the Frobenius automorphism for the relevant idempotent Frobenius system is always trivial, which is not the case). This covers a large part of Theorem 5.1. However, the antipode is used in [5], while it is not assumed to exist in Theorem 5.1. On the other hand the part of the antipode relevant for the proof (its restrictions to the source and target counital subalgebras) is present in any weak bialgebra, even if it does not possess an antipode; this was proved by Nill [15] along with the fact that the counital subalgebras have an idempotent Frobenius system.

After providing some definitions in Sections 2 and 3, we start the real work in Section 4 by proving some basic facts about weak bialgebras; notably we construct an idempotent Frobenius system for the counital subalgebra of a weak bialgebra is. As we acknowledged already, this (and all the facts proved in Section 4) can be found in the literature. However, Etingof and Nikshych [5] use antipodes, while Nill's paper [15] consistently uses the 
assumption that the weak bialgebra in question is finite-dimensional. The same general assumption is used in many places in [1]. Instead of examining the proofs in each situation to convince the reader that the extra assumptions are not necessary, it seemed easier and more useful to develop the basic facts that we need from scratch.

In Section 5 we prove that any weak bialgebra $H$ is a $\times_{R}$-bialgebra (which is $[5$, Prop. 2.3.1] if $H$ is a weak Hopf algebra), and conversely, that any $\times_{R}$-bialgebra with separable $R$ is a weak bialgebra.

In Section 6 we adress the question when a weak bialgebra is a weak Hopf algebra. We show in Theorem 6.1 that a weak Hopf algebra can be characterized as a weak bialgebra $H$ for which a certain canonical map $H \otimes_{H_{t}} H \rightarrow \Delta(1)(H \otimes H)$ is a bijection; this is analogous to a well-known characterization of ordinary Hopf algebras. (We should note that certain identities for antipodes useful for proving one of the implications in Theorem 6.1 can be found in [1], again under different assumptions). This also proves that a weak bialgebra is a weak Hopf algebra if and only if the associated $\times_{R}$-bialgebra is a $\times_{R}$-Hopf algebra in the sense of the definition we have given in [18]. One should note that this is in general rather different from the definition of a Hopf algebroid by Lu, which involves an antipodal anti-algebra map and a certain splitting of the epimorphism $H \otimes H \rightarrow H \otimes_{R} H$. Our definition by bijectivity of a canonical map has the advantage of having a canonical characterization in terms of properties of the module category of $H$.

In Section 7 we show that the correspondence between weak bialgebras and $\times_{R^{-}}$ bialgebras is compatible with taking duals (in the finite-dimensional case), and with taking the respective comodule categories.

2. $\times_{R}$-bialgebras. In this section we will recall the necessary definitions and notations on $\times_{R}$-bialgebras. For more details we refer to $[19,20,16]$.

Throughout the paper, $k$ denotes a base field. Modules, algebras, unadorned tensor products etc. are understood to be over $k$ if nothing else is indicated.

Let $R$ be a $k$-algebra. We denote the opposite algebra by $\bar{R}$, we let $R \ni r \mapsto \bar{r} \in \bar{R}$ denote the obvious $k$-algebra antiisomorphism, and abbreviate the enveloping algebra $R^{e}:=R \otimes \bar{R}$. We write $r \bar{s}:=r \otimes \bar{s} \in R \otimes \bar{R}$ for $r, s \in R$.

For $M, N \in R^{e} \mathcal{M}$ we let

$$
\int_{r}{ }_{r} M \otimes{ }_{r} N:=M \otimes N /\langle\bar{r} m \otimes n-m \otimes r n \mid r \in R, m \in M, n \in N\rangle
$$

and we let $\int^{r}{ }_{r} M \otimes{ }_{r} N \subset M \otimes N$ denote the $k$-submodule consisting of all elements $\sum m_{i} \otimes n_{i} \in M \otimes N$ satisfying $\sum \bar{r} m_{i} \otimes n_{i}=\sum m_{i} \otimes r n_{i}$ for all $r \in R$. Variations of the $\int_{r}$ and $\int^{r}$ notations, which are due to MacLane, will be used without further notice. We abbreviate $\int_{r} \bar{r} M \otimes_{r} N=M \diamond_{R} N$ for $M, N \in R^{e} \mathcal{M}$.

For two $R^{e}$-bimodules $M$ and $N$ we let

$$
M \times{ }_{R} N:=\int^{s} \int_{r} M_{\bar{s}} \otimes{ }_{r} N_{s} .
$$

If $M, N$ are $R^{e}$-rings, then so is $M \times_{R} N$, with multiplication given by $\left(\sum m_{i} \otimes n_{i}\right)\left(\sum m_{j}^{\prime} \otimes\right.$ $\left.n_{j}^{\prime}\right)=\sum m_{i} m_{j}^{\prime} \otimes n_{i} n_{j}^{\prime}$, and $R^{e}$-ring structure

$$
R^{e} \ni r \otimes \bar{s} \mapsto r \otimes \bar{s} \in M \times_{R} N
$$


For $M, N, P \in R^{e} \mathcal{M}_{R^{e}}$ one defines

$$
M \times_{R} P \times_{R} N:=\int^{s, u} \int_{r, t}{ }_{r} M_{\bar{s}} \otimes{ }_{r, \bar{t}} P_{s, \bar{u}} \otimes{ }_{t} N_{u}
$$

(where $\int^{s, u}:=\int^{s} \int^{u}=\int^{u} \int^{s}$ ). There are associativity maps

$$
\begin{aligned}
& \left(M \times_{R} P\right) \times_{R} N \stackrel{\alpha}{\rightarrow} M \times_{R} P \times_{R} N, \\
& M \times_{R}\left(P \times_{R} N\right) \stackrel{\alpha^{\prime}}{\rightarrow} M \times_{R} P \times_{R} N,
\end{aligned}
$$

given on elements by the obvious formulas (doing nothing), but which need not be isomorphisms. If $M, N$ and $P$ are $R^{e}$-rings, so is $M \times_{R} N \times_{R} P$, and $\alpha, \alpha^{\prime}$ are $R^{e}$-ring maps.

An $R^{e}$-ring structure on the algebra $\mathrm{E}=\operatorname{End}(R)$ is given by $r \otimes \bar{s} \mapsto(t \mapsto r t s)$. We have, for any $M \in R^{e} \mathcal{M}_{R^{e}}$, two $R^{e}$-bimodule maps

$$
\begin{gathered}
\theta: M \times_{R} \operatorname{End}(R) \rightarrow M, \quad m \otimes f \mapsto \overline{f(1)} m, \\
\theta^{\prime}: \operatorname{End}(R) \times_{R} M \rightarrow M, \quad f \otimes m \mapsto f(1) m,
\end{gathered}
$$

which are $R^{e}$-ring homomorphisms if $M$ is an $R^{e}$-ring.

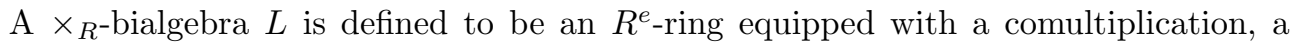
map $\Gamma: L \rightarrow L \times_{R} L$ of $R^{e}$-rings over $R^{e}$, and a counit, a map $C: L \rightarrow \mathrm{E}$ of $R^{e}$-rings, such that

$$
\begin{gathered}
\alpha\left(\Gamma \times{ }_{R} L\right) \Gamma=\alpha^{\prime}\left(L \times{ }_{R} \Gamma\right) \Gamma: L \rightarrow L \times{ }_{R} L \times{ }_{R} L, \\
\theta\left(L \times{ }_{R} C\right) \Gamma=\operatorname{id}_{L}=\theta\left(C \times{ }_{R} L\right) \Gamma .
\end{gathered}
$$

Note that an $R^{e}$-ring map $\Gamma: L \rightarrow L \times_{R} L$ induces a map $\Gamma_{0}: L \rightarrow L \diamond L$ in $R^{e} \mathcal{M}$, and an $R^{e}$-ring map $C: L \rightarrow$ E induces a map $C_{0}: L \ni \ell \mapsto C(\ell)(1) \in R$ in $R^{e} \mathcal{M}$. One checks that $\Gamma$ and $C$ fulfill the equations (2.1) and (2.2) if and only if

$$
\left(\Gamma_{0} \diamond L\right) \Gamma_{0}=\left(L \diamond \Gamma_{0}\right) \Gamma_{0}: L \rightarrow L \diamond L \diamond L
$$

and $\left(C_{0} \diamond L\right) \Gamma_{0}=\operatorname{id}_{L}=\left(L \diamond C_{0}\right)$ hold. These mean that $L$, considered as an $R$ - $R$-bimodule via the left $R^{e}$-module structure, is an $R$-coring.

For $\times_{R}$-bialgebras we will make use of the variants $\Gamma(\ell)=: \ell_{[1]} \otimes \ell_{[2]} \in L \times_{R} L$ and

$$
\alpha\left(\Gamma \times_{R} L\right) \Gamma(\ell)=: \ell_{[1]} \otimes \ell_{[2]} \otimes \ell_{[3]} \in L \times_{R} L \times_{R} L .
$$

of usual Sweedler notation (reserving $\ell_{(1)} \otimes \ell_{(2)}$ for usual coalgebra structures).

If $L$ is a $\times{ }_{R}$-bialgebra, then the tensor product $M \diamond_{R} N$ of $M, N \in{ }_{L} \mathcal{M}$ can be endowed with an $L$-module structure by the usual formula $\ell(m \otimes n)=\ell_{[1]} m \otimes \ell_{[2]} n$.

The suitable definition of comodules over a $\times_{R}$-bialgebra $L$ is as follows: A left $L$ comodule is an $R$-bimodule $M$ together with a map $\lambda: M \rightarrow L \times_{R} M$ of $R$-bimodules such that

$$
\alpha^{\prime}\left(L \times_{R} \lambda\right) \lambda=\alpha\left(\Gamma \times_{R} M\right) \lambda: M \rightarrow L \times_{R} L \times_{R} M
$$

and $\theta^{\prime}\left(C \times_{R} M\right) \lambda=\operatorname{id}_{M}$ hold. If we denote by $\lambda_{0}: M \rightarrow L \diamond M$ the composition of $\lambda$ with the inclusion of $L \diamond M$ into $L \times_{R} M$, then coassociativity is equivalent to $\left(L \diamond \lambda_{0}\right) \lambda_{0}=$ $\left(\Gamma_{0} \diamond M\right) \lambda_{0}: M \rightarrow L \diamond L \diamond M$ and $\left(C_{0} \diamond M\right) \lambda_{0}=\operatorname{id}_{M}$. We will denote by ${ }^{L} \mathcal{M}$ the category of left $L$-comodules. We will use Sweedler notation in the form $\lambda(m)=m_{[-1]} \otimes m_{[0]}$ and $\alpha\left(\Gamma \times_{R} M\right)(m)=m_{[-2]} \otimes m_{[-1]} \otimes m_{[0]}$ for $L$-comodules. 
The category ${ }^{L} \mathcal{M}$ of left $L$-comodules over a $\times_{R}$-bialgebra is monoidal. The tensor product of $M, N \in{ }^{L} \mathcal{M}$ is their tensor product $M \otimes_{R} N$ over $R$, equipped with the comodule structure

$$
\begin{aligned}
M \underset{R}{\otimes} N & \rightarrow L \times_{R}(M \underset{R}{\otimes} N), \\
m \otimes n & \mapsto m_{[-1]} n_{[-1]} \otimes m_{[0]} \otimes n_{[0]} .
\end{aligned}
$$

3. Separable algebras and Frobenius systems. In this section we compile a few facts and notations on separable and Frobenius algebras. All of the material in this section (except for a recent result of Kadison and Szlachányi) is certainly folklore.

Let $R$ be a $k$-algebra. Recall that $R$ is Frobenius if there is a Frobenius system $(\phi, e)$ for $R$, which in turn consists by definition of a $k$-linear map $\phi: R \rightarrow k$, and an element $e=e^{(1)} \otimes e^{(2)} \in R \otimes R$ such that

$$
\forall r \in R: r=\phi\left(r e^{(1)}\right) e^{(2)}=e^{(1)} \phi\left(e^{(2)} r\right) .
$$

Equivalently, $R$ is finite-dimensional, and there is a $k$-linear map $\phi: R \rightarrow k$ such that the bilinear form $B_{\phi}: R \times R \rightarrow k$ given by $B_{\phi}(x, y)=\phi(x y)$ is nondegenerate. It follows that $e \in R \otimes R$ is a Casimir element in the sense that $(x \otimes 1) e=e(1 \otimes x)$ in the algebra $R \otimes R$ for each $x \in R$ : by nondegeneracy of $B_{\phi}$ it is sufficient to observe $\phi\left(y x e^{(1)}\right) e^{(2)}=$ $y x=\phi\left(y e^{(1)}\right) e^{(2)} x$ for all $y \in R$. Recall that the Frobenius automorphism $\theta: R \rightarrow R$ defined by the Frobenius system $(\phi, e)$ is by definition the linear map $\theta: R \rightarrow R$ with $\phi(x y)=\phi(y \theta(x))$ for all $x, y \in R$. It is an algebra automorphism. We have $(1 \otimes x) e=$ $e(\theta(x) \otimes 1)$ in $R \otimes R$ for all $x \in R$, by the calculation $\phi\left(y e^{(1)} \theta(x)\right) e^{(2)}=\phi\left(x y e^{(1)}\right) e^{(2)}=$ $x y=\phi\left(y e^{(1)}\right) x e^{(2)}$. It is easy to see that this property characterizes $\theta$, so that $(\phi, e)$ is a symmetric Frobenius system (i.e. $B_{\phi}$ is symmetric) if and only if $\theta$ is the identity if and only if $\left(\phi, e^{(2)} \otimes e^{(1)}\right)$ is a Frobenius system if and only if $e^{(2)} \otimes e^{(1)}=e$.

If $(\phi, e)$ is a Frobenius system, and $t \in R$ is invertible, then $(\psi, f)$ defined by $\psi(x)=$ $\phi(t x)$ and $f=\left(1 \otimes t^{-1}\right) e$, is also a Frobenius system by the calculations $\psi\left(x f^{(1)}\right) f^{(2)}=$ $\phi\left(t x e^{(1)}\right) t^{-1} e^{(2)}=t^{-1} t x=x$ and $f^{(1)} \psi\left(f^{(2)} x\right)=e^{(1)} \phi\left(t t^{-1} e^{(2)} x\right)=e^{(1)} \phi\left(e^{(2)} x\right)=x$.

Conversely, if $(\psi, f)$ is another Frobenius system, define $t:=\psi\left(e^{(1)}\right) e^{(2)}$. Then $\phi(t x)=$ $\psi\left(e^{(1)}\right) \phi\left(e^{(2)} x\right)=\psi(x)$ for all $x \in R$, further $e=(1 \otimes t) f$ since $f^{(1)} \phi\left(t f^{(2)} x\right)=$ $f^{(1)} \psi\left(f^{(2)} x\right)=x=e^{(1)} \phi\left(e^{(2)} x\right)$ for all $x \in R$. Finally $t$ is invertible with inverse $\phi\left(f^{(1)}\right) f^{(2)}$ since $\phi\left(f^{(1)}\right) f^{(2)} t=\phi\left(t f^{(1)}\right) f^{(2)}=\psi\left(f^{(1)}\right) f^{(2)}=1$ and $t \phi\left(f^{(1)}\right) f^{(2)}=$ $\phi\left(e^{(1)}\right) e^{(2)}=1$.

Let $(\phi, e)$ be a Frobenius system. Then $e$ is a separability idempotent for $R$ if and only if $\nabla(e)=1$, in which case we say that $(\phi, e)$ is an idempotent Frobenius system. If $R$ is a commutative separable $k$-algebra with separability idempotent $e$, and $\phi: R \rightarrow k$ is the trace functional, then $(\phi, e)$ is an idempotent Frobenius system. To see this it suffices to treat the case where $R$ is a field. Let $e$ be a separability idempotent, and write $e=\sum_{i=1}^{n} x_{i} \otimes y_{i}$ with $n$ minimal. Then the elements $x_{i}$ generate $R$ as a $k$-space (hence they are a basis). For take any $x \in R$, put $t:=x_{1}^{-1} x$, and consider $\phi \in R^{*}$ with $\varphi\left(y_{i}\right)=\delta_{1, i}$. Then

$$
x=(\mathrm{id} \otimes \varphi)\left(\sum t x_{i} \otimes y_{i}\right)=\sum x_{i} \varphi\left(y_{i} t\right)
$$

Similarly the $y_{i}$ form a basis of $R$. Now let $\phi(r)$ be the trace of multiplication by $r$ as 
an endomorphism of $R$; this defines $\phi: R \rightarrow k$, and we claim that $(\phi, e)$ is an idempotent Frobenius system. Let $\left(y^{i}\right)$ be the dual basis of $\left(y_{i}\right)$. Then $\phi(r)=\sum y^{i}\left(r y_{i}\right)$, so that

$$
\phi\left(x e^{(1)}\right) e^{(2)}=\sum \phi\left(x x_{i}\right) y_{i}=\sum x^{j}\left(x x_{j} x_{i}\right) y_{i} x=\sum x^{j}\left(x_{i}\right) y_{i} x x_{j}=\sum y_{j} x x_{j}=x
$$

follows for $x \in R$. Similarly $e^{(1)} \phi\left(e^{(2)} x\right)=x$. Much more generally, Kadison and Szlachányi [9, Prop. 9.3] have shown that every separable $k$-algebra has an idempotent Frobenius system. However, if $R$ is symmetric and separable, it may not be possible to have a symmetric idempotent Frobenius system; an example is the matrix algebra $M_{p}(k)$ for a field $k$ of characteristic $p>0$.

If $(\phi, e)$ and $(\psi, f)$ are two idempotent Frobenius systems, then there is an invertible $t \in R$ with $e^{(1)} t^{-1} e^{(2)}=1$, such that $\psi(x)=\phi(t x)$ for all $x$ and $f=\left(1 \otimes t^{-1}\right) e$.

4. Weak Hopf algebras. A weak bialgebra $H=(H, \nabla, \Delta)$ is by definition an algebra and coalgebra $H$ such that the comultiplication $\Delta: H \rightarrow H \otimes H$ is multiplicative, and the following four conditions hold for all $f, g, h \in H$ :

$$
\begin{gathered}
\varepsilon(f g h)=\varepsilon\left(f g_{(1)}\right) \varepsilon\left(g_{(2)} h\right), \\
\varepsilon(f g h)=\varepsilon\left(f g_{(2)}\right) \varepsilon\left(g_{(1)} h\right), \\
1_{(1)} \otimes 1_{(2)} \otimes 1_{(3)}=(\Delta(1) \otimes 1)(1 \otimes \Delta(1)), \\
1_{(1)} \otimes 1_{(2)} \otimes 1_{(3)}=(1 \otimes \Delta(1))(\Delta(1) \otimes 1) .
\end{gathered}
$$

These four conditions weaken the conditions of multiplicativity of the counit, and comultiplicativity of the unit, which are not required in a weak bialgebra. Note that by the symmetries of the definition, the opposite $H^{\mathrm{op}}$, coopposite $H^{\mathrm{cop}}$, and the opposite and coopposite (or biopposite) $H^{\text {bop }}$ are weak bialgebras as well.

We define the source and target counital maps $\varepsilon_{s, t}: H \rightarrow H$ of a weak bialgebra $H$ to be

$$
\varepsilon_{s}(h)=1_{(1)} \varepsilon\left(h 1_{(2)}\right), \quad \varepsilon_{t}(h)=\varepsilon\left(1_{(1)} h\right) 1_{(2)} .
$$

And denote their images by $H_{s, t}:=\varepsilon_{s, t}(H)$; these are called the source and target counital subalgebras (see below) of $H$. We note the variants $\varepsilon_{s, t}^{\prime}$ with

$$
\varepsilon_{s}^{\prime}(h)=1_{(1)} \varepsilon\left(1_{(2)} h\right), \quad \varepsilon_{t}^{\prime}(h)=\varepsilon\left(h 1_{(1)}\right) 1_{(2)} .
$$

Obviously these are the source and target counital maps for the weak bialgebra $H^{\text {op }}$, which means that general statements on them will follow from general statements on $\varepsilon_{s, t}$ mutatis mutandis. We'll use in the same way that $\varepsilon_{s}$ is the target counital map of $H^{\text {bop }}$.

Note $h_{(1)} \varepsilon_{s}\left(h_{(2)}\right)=h_{(1)} 1_{(1)} \varepsilon\left(h_{(2)} 1_{(2)}\right)=h$, so also

$$
h_{(1)} \varepsilon_{s}\left(h_{(2)}\right)=\varepsilon_{t}\left(h_{(1)}\right) h_{(2)}=\varepsilon_{s}^{\prime}\left(h_{(2)}\right) h_{(1)}=h_{(2)} \varepsilon_{t}^{\prime}\left(h_{(1)}\right)=h
$$

for all $h \in H$. Moreover

$$
\varepsilon_{t}\left(1_{(1)} h\right) 1_{(2)}=\varepsilon\left(1_{(1)}^{\prime} 1_{(1)} h\right) 1_{(2)}^{\prime} 1_{(2)}=\varepsilon_{t}(h)
$$

We have

$$
1_{(1)} \otimes \varepsilon_{t}\left(1_{(2)}\right)=1_{(1)} \otimes \varepsilon\left(1^{\prime}{ }_{(1)} 1_{(2)}\right) 1_{(2)}^{\prime}=1_{(1)} \otimes \varepsilon\left(1_{(2)}\right) 1_{(3)}=1_{(1)} \otimes 1_{(2)}
$$

hence

$$
1_{(1)} \otimes 1_{(2)}=\varepsilon_{s}\left(1_{(1)}\right) \otimes 1_{(2)}=\varepsilon_{s}\left(1_{(1)}\right) \otimes \varepsilon_{t}\left(1_{(2)}\right)
$$


and the same identities with $\varepsilon_{s, t}$ replaced by $\varepsilon_{s, t}^{\prime}$. In particular $\Delta(1) \in H_{s} \otimes H_{t}$. It also follows that $\varepsilon_{s, t}(x)=\varepsilon_{s, t}^{\prime}(x)=x$ for all $x \in H_{s, t}$, so that $\varepsilon_{t}, \varepsilon_{t}^{\prime}$ are idempotent projectors onto $H_{t}$.

The calculation

$$
\Delta\left(\varepsilon_{t}(x)\right)=\varepsilon\left(1_{(1)} h\right) 1_{(2)} \otimes 1_{(3)}=\varepsilon\left(1_{(1)} h\right) 1_{(2)} 1_{(1)}^{\prime} \otimes 1_{(2)}^{\prime}=\varepsilon_{t}(h) 1_{(1)} \otimes 1_{(2)}
$$

for all $h \in H$ shows the first part of

$$
\forall x \in H_{t}: \Delta(x)=x 1_{(1)} \otimes 1_{(2)}=1_{(1)} x \otimes 1_{(2)},
$$

the second is proved similarly, and as a corollary we have

$$
\forall x \in H_{s}: \Delta(x)=1_{(1)} \otimes x 1_{(2)}=1_{(1)} \otimes 1_{(2)} x .
$$

For all $g, h \in H$ we have

$$
\varepsilon(g h)=\varepsilon\left(g 1_{(2)}\right) \varepsilon\left(1_{(1)} h\right)=\varepsilon\left(g \varepsilon_{t}(h)\right)
$$

by (4.2), hence

$$
\varepsilon_{t}(g h)=\varepsilon\left(1_{(1)} g h\right) 1_{(2)}=\varepsilon\left(1_{(1)} g \varepsilon_{t}(h)\right) 1_{(2)}=\varepsilon_{t}\left(g \varepsilon_{t}(h)\right),
$$

and further

$$
\left.g \varepsilon_{t}(h)=\varepsilon_{t}\left(g_{(1)} \varepsilon_{t}(h)_{(1)}\right)\right) g_{(2)} \varepsilon_{t}(h)_{(2)}=\varepsilon_{t}\left(g_{(1)} \varepsilon_{t}(h)\right) g_{(2)}=\varepsilon_{t}\left(g_{(1)} h\right) g_{(2)}
$$

hence

$$
\forall x \in H_{t} \forall h \in H: x \varepsilon_{t}(h)=\varepsilon_{t}\left(1_{(1)} x h\right) 1_{(2)}=\varepsilon_{t}(x h) .
$$

In particular $H_{t}$ is multiplicatively closed; it is a subalgebra because also $\varepsilon_{t}(1)=$ $\varepsilon\left(1^{\prime}{ }_{(1)} 1\right) 1^{\prime}{ }_{(2)}=\varepsilon\left(1^{\prime}{ }_{(1)}\right) 1^{\prime}{ }_{(2)}=1$.

For $g, h \in H$ we have

$$
\varepsilon_{t}(g) \varepsilon_{s}(h)=\varepsilon\left(1_{(1)} g\right) 1_{(2)} 1^{\prime}{ }_{(1)} \varepsilon\left(h 1^{\prime}{ }_{(2)}\right)=\varepsilon\left(1_{(1)} g\right) 1^{\prime}{ }_{(1)} 1_{(2)} \varepsilon\left(h 1^{\prime}{ }_{(2)}\right)=\varepsilon_{s}(h) \varepsilon_{t}(g),
$$

so that the subalgebras $H_{s}$ and $H_{t}$ commute element-wise.

LEMMA 4.1. Let $H$ be a weak bialgebra. The target counital map $\varepsilon_{t}$ induces an algebra antiisomorphism $H_{s} \rightarrow H_{t}$, whose inverse is induced by $\varepsilon_{s}^{\prime}$.

Proof. To see that $\varepsilon_{t}$ is an an anti-algebra map we compute more generally

$$
\varepsilon_{t}(y h)=\varepsilon_{t}\left(y \varepsilon_{t}(h)\right)=\varepsilon_{t}\left(\varepsilon_{t}(h) y\right)=\varepsilon_{t}(h) \varepsilon_{t}(y)
$$

for all $y \in H_{s}$ and $h \in H$, using (4.9) and (4.10).

To prove $\varepsilon_{s}^{\prime}$ induces an inverse isomorphism to the map induced by $\varepsilon_{t}$, we use that more generally

$$
\forall h \in H: \varepsilon_{t} \varepsilon_{s}^{\prime}(h)=\varepsilon_{t}(h)
$$

by the calculation

$$
\varepsilon_{t} \varepsilon_{s}^{\prime}(h)=\varepsilon\left(1_{(1)} \varepsilon_{s}^{\prime}(h)\right) 1_{(2)}=\varepsilon\left(1_{(1)} 1^{\prime}(1) \varepsilon\left(1^{\prime}(2) h\right)\right) 1_{(2)} \stackrel{(4.1)}{=} \varepsilon\left(1_{(1)} h\right) 1_{(2)}=\varepsilon_{t}(h)
$$

Applying this to $H^{\text {bop }}$ yields $\varepsilon_{s}^{\prime} \varepsilon_{t}(h)=\varepsilon_{s}^{\prime}(h)$ for all $h \in H$, and this taken together with (4.11) proves the claim. 
Proposition 4.2. Let $H$ be a weak bialgebra. Then the target counital subalgebra $H_{t}$ is separable with idempotent Frobenius system

$$
\left(\left.\varepsilon\right|_{H_{t}},\left(\varepsilon_{t} \otimes H\right) \Delta(1)\right) .
$$

Proof. The claimed idempotent Frobenius system is given more explicitly by

$$
e=\varepsilon_{t}\left(1_{(1)}\right) \otimes 1_{(2)}=\varepsilon\left(1_{(1)}^{\prime} 1_{(1)}\right) 1_{(2)}^{\prime} \otimes 1_{(2)} .
$$

We have, for all $x \in H_{t}$ :

$$
\varepsilon\left(x e^{(1)}\right) e^{(2)}=\varepsilon\left(1^{\prime}{ }_{(1)} 1_{(1)}\right) \varepsilon\left(x 1^{\prime}{ }_{(2)}\right) 1_{(2)} \stackrel{(4.4)}{=} \varepsilon\left(x 1_{(1)}\right) 1_{(2)}=\varepsilon_{t}^{\prime}(x)=x
$$

and

$$
e^{(1)} \varepsilon\left(e^{(2)} x\right)=\varepsilon\left(1^{\prime}{ }_{(1)} 1_{(1)}\right) 1^{\prime}{ }_{(2)} \varepsilon\left(1_{(2)} x\right) \stackrel{(4.3)}{=} \varepsilon\left(1^{\prime}{ }_{(1)} x\right) 1^{\prime}{ }_{(2)}=\varepsilon_{t}(x)=x
$$

while $\nabla(e)=1$ is quite obvious.

It follows that

$$
\forall x \in H_{t}: x \varepsilon_{t}\left(1_{(1)}\right) \otimes 1_{(2)}=\varepsilon_{t}\left(1_{(1)}\right) \otimes 1_{(2)} x .
$$

Applying the Lemma to $H^{\text {bop }}$ yields that $H_{s}^{\text {op }}$ is separable with idempotent Frobenius system $\left(\varepsilon, 1_{(1)} \otimes \varepsilon_{s}\left(1_{(2)}\right)\right)$. In particular

$$
\forall y \in H_{s}: 1_{(1)} y \otimes \varepsilon_{s}\left(1_{(2)}\right)=1_{(1)} \otimes y \varepsilon_{s}\left(1_{(2)}\right)
$$

Applying $\varepsilon_{s}^{\prime}$ (which is an anti-algebra map restricted to $H_{t}$ ) to the first tensor factor of (4.12), we obtain

$$
\forall x \in H_{t}: 1_{(1)} \varepsilon_{s}^{\prime}(x) \otimes 1_{(2)}=1_{(1)} \otimes 1_{(2)} x
$$

\section{Weak bialgebras are $\times_{R}$-bialgebras}

Theorem 5.1. Let $(H, \Delta, \varepsilon)$ be a weak bialgebra. Put $R:=H_{t}$. Then the structure $(H, \Gamma, C)$ of a $\times_{R}$-bialgebra on $H$ is given as follows: The $R^{e}$-ring structure of $H$ is given by $\iota(x \otimes \bar{y})=x \varepsilon_{s}^{\prime}(y)$, the comultiplication

$$
\Gamma: H \rightarrow H \times{ }_{R} H \subset H \diamond H
$$

is the composition of $\Delta$ with the canonical surjection $H \otimes H \rightarrow H \diamond H$. The counit is

$$
C: H \ni h \mapsto\left(x \mapsto \varepsilon_{t}(h x)\right) \in \operatorname{End}(R) .
$$

Proof. $H$ is an $R^{e}$-ring as claimed since $\varepsilon_{s}^{\prime}$ induces an antiisomorphism of $H_{t}$ with $H_{s}$, and $H_{s}$ and $H_{t}$ commute element-wise.

That $\Gamma_{0}: H \rightarrow H \otimes H \rightarrow H \diamond H$ takes values in $H \times_{R} H$ follows from

$$
\Gamma(h)=\Gamma(h \cdot 1)=h_{(1)} 1_{(1)} \otimes h_{(2)} 1_{(2)}
$$

and (4.14).

It is clear that $\Gamma$ is an algebra map, since $\Delta$ is multiplicative and $\Gamma(1)=1$ in $H \diamond H$. Also, $\Gamma$ is a map of $R^{e}$-rings by (4.7) and (4.6), and obviously coassociative since $\Delta$ is.

The map $C$ is unit-preserving since $\varepsilon_{t}$ is idempotent, and multiplicative since

$$
C(g) C(h)(x)=C(g)\left(\varepsilon_{t}(h x)\right)=\varepsilon_{t}\left(g \varepsilon_{t}(h x)\right)=\varepsilon_{t}(g h x)
$$

for all $g, h \in H$ and $x \in H_{t}$, using (4.9). Moreover $C(y)(x)=\varepsilon_{t}(y x)=y x$ and $C(\bar{y})(x)=$ $\varepsilon_{t}\left(\varepsilon_{s}^{\prime}(y) x\right)=\varepsilon_{t}\left(x \varepsilon_{s}^{\prime}(y)\right)=x \varepsilon_{t} \varepsilon_{s}^{\prime}(y)=x y$ for $x, y \in H_{t}$ show that $C$ is a map of $R^{e}$-rings. 
It remains to check that $C$ is a counit: We have

$$
C\left(h_{[1]}\right)(1) h_{[2]}=\varepsilon\left(1_{(1)} h_{[1]}\right) 1_{(2)} h_{[2]}=\varepsilon\left(1_{(1)} h_{(1)}\right) 1_{(2)} h_{(2)}=h
$$

as well as

$$
\begin{aligned}
\overline{C\left(h_{[2]}\right)(1)} h_{[1]} & =\overline{\varepsilon\left(1_{(1)} h_{[2]}\right) 1_{(2)}} h_{[1]}=\varepsilon\left(1_{(1)} h_{(2)}\right) \varepsilon_{s}^{\prime}\left(1_{(2)}\right) h_{(1)} \\
& =\varepsilon\left(1_{(1)} h_{(2)}\right) 1^{\prime}{ }_{(1)} \varepsilon\left(1^{\prime}{ }_{(2)} 1_{(2)}\right) h_{(1)} \stackrel{(4.1)}{=} \varepsilon\left(1^{\prime}{ }_{(2)} h_{(2)}\right) 1^{\prime}{ }_{(1)} h_{(1)}=h
\end{aligned}
$$

for all $h \in H$.

The theorem above (which is [5, Prop. 2.3.1] in the case where $H$ is a weak Hopf algebra) shows that any weak bialgebra is a $\times_{R}$-bialgebra in which, by Proposition $4.2, R$ is separable. We will also prove a converse Theorem 5.5. Just as in the case of commutative separable $R$ treated in [17], this is based on the following simple observation:

REMARK 5.2. Let $R$ be a separable algebra with separability idempotent $e$. Then for $M \in \mathcal{M}_{R}$ and $N \in{ }_{R} \mathcal{M}$ the identity on $M \otimes N$ induces an isomorphism

$$
\gamma: M e^{(1)} \otimes e^{(2)} N \rightarrow M \underset{R}{\otimes} N
$$

with inverse given by $\gamma^{-1}(m \otimes n)=m e^{(1)} \otimes e^{(2)} n$.

Before using this (implicitly) to prove Theorem 5.5, we will use it to compare the tensor product defined on modules over a weak bialgebra by Böhm and Szlachányi [3] with the tensor product defined on the modules over the corresponding $\times_{R}$-bialgebra. The tensor product on $H$-modules for a weak bialgebra $H$ is given by $M \odot N:=\Delta(1)(M \otimes N)$ for $M, N \in{ }_{H} \mathcal{M}$, with the diagonal left $H$-module structure induced via $\Delta$.

Proposition 5.3. Let $H$ be a weak bialgebra. Then the isomorphisms

$$
\gamma=\gamma_{M N}: M \odot N \rightarrow M \diamond N
$$

for $M, N \in{ }_{H} \mathcal{M}$ endow the identity functor with the structure of a monoidal functor

$$
(I d, \gamma):\left({ }_{H} \mathcal{M}, \diamond\right) \rightarrow\left({ }_{H} \mathcal{M}, \odot\right)
$$

Proof. The idempotent Frobenius system we have found for $R=H_{t}$ in Proposition 4.2 is such that $\overline{e^{(1)}} \otimes e^{(2)}=\Delta(1)$. Thus $\gamma$ is a vector space isomorphism by Remark 5.2 ; it is linear by definition of comultiplication in the $\times_{R}$-bialgebra associated to the weak bialgebra $H$. Coherence of the monoidal functor is evident since $\gamma$ is induced by the identity (and we skip treating unit objects altogether).

Remark 5.4. The arguments used in Proposition 5.3 could be rewritten to be a different proof of Theorem 5.1: A weak bialgebra $H$ is an $R^{e}$-ring for $R=R_{t}$; since $R$ is separable, we can use Remark 5.2 to endow the underlying functor ${ }_{H} \mathcal{M} \rightarrow R^{e} \mathcal{M}$ with the structure of a monoidal functor. It then follows from [16, Thm.5.1] that $H$ has a $\times_{R}$-bialgebra structure.

We now proceed to prove the converse of Theorem 5.1:

ThEOREM 5.5. Let $R$ be a separable algebra with idempotent Frobenius system $(\phi, e)$. Let $(H, \Gamma, C)$ be a $\times_{R}$-bialgebra. Then the structure $(H, \Delta, \varepsilon)$ of a weak bialgebra on $H$ 
is given by

$$
\Delta(h)=\sum \overline{e^{(1)}} h_{[1]} \otimes e^{(2)} h_{[2]}, \quad \varepsilon(h)=\phi(C(h)(1)) .
$$

Proof. The map $\Delta$ is well-defined since

$$
f: H \diamond H \ni g \otimes h \mapsto \overline{e^{(1)}} g \otimes e^{(2)} h \in H \otimes H
$$

is well-defined, since $\overline{e^{(1)}} \bar{x} g \otimes e^{(2)} h=\overline{x e^{(1)}} g \otimes e^{(2)} h=\overline{e^{(1)}} g \otimes e^{(2)} x h$ holds for all $g, h \in H$ and $x \in R$. We have

$$
\begin{aligned}
\Delta\left(h_{(1)}\right) \otimes h_{(2)} & =\overline{e^{(1)}}\left(\overline{\tilde{e}^{(1)}} h_{[1]}\right)_{[1]} \otimes e^{(2)}\left(\overline{\tilde{e}^{(1)}} h_{[1]}\right)_{[2]} \otimes \tilde{e}^{(2)} h_{[2]} \\
& =\overline{e^{(1)}} h_{[1][1]} \otimes e^{(2)} \overline{\tilde{e}^{(1)}} h_{[1][2]} \otimes \tilde{e}^{(2)} h_{[2]}=\overline{e^{(1)}} h_{[1]} \otimes e^{(2)} \overline{\tilde{e}^{(1)}} h_{[2][1]} \otimes \tilde{e}^{(2)} h_{[2][2]} \\
& =\overline{e^{(1)}} h_{[1]} \otimes \overline{\tilde{e}^{(1)}}\left(e^{(2)} h_{[2]}\right)_{[1]} \otimes \tilde{e}^{(2)}\left(e^{(2)} h_{[2]}\right)_{[2]}=h_{(1)} \otimes \Delta\left(h_{(2)}\right)
\end{aligned}
$$

showing that $\Delta$ is coassociative. The map $\varepsilon$ is a counit since

$$
h_{(1)} \varepsilon\left(h_{(2)}\right)=\overline{e^{(1)}} h_{[1]} \phi\left(C\left(e^{(2)} h_{[2]}\right)(1)\right)=\overline{e^{(1)}} h_{[1]} \phi\left(e^{(2)} C\left(h_{[2]}\right)(1)\right)=\overline{C\left(h_{[2]}\right)(1)} h_{[1]}=h
$$

and

$$
\varepsilon\left(h_{(1)}\right) h_{(2)}=\phi\left(C\left(\overline{e^{(1)}} h_{[1]}\right)(1)\right) e^{(2)} h_{[2]}=\phi\left(C\left(h_{[1]}\right)(1) e^{(1)}\right) e^{(2)} h_{[2]}=C\left(h_{[1]}\right)(1) h_{[2]}=h .
$$

$\Delta$ is multiplicative by the calculation

$$
\begin{aligned}
\Delta(g) \Delta(h) & =\overline{e^{(1)}} g_{[1]} \overline{\tilde{e}^{(1)}} h_{[1]} \otimes e^{(2)} g_{[2]} \tilde{e}^{(2)} h_{[2]}=\overline{e^{(1)}} g_{[1]} h_{[1]} \otimes e^{(2)} \tilde{e}^{(1)} \tilde{e}^{(2)} h_{[2]} \\
& =\overline{e^{(1)}} g_{[1]} h_{[1]} \otimes e^{(2)} g_{[2]} h_{[2]}=\Delta(g h)
\end{aligned}
$$

for all $g, h \in H$, using $\Gamma(g) \in H \times{ }_{R} H$.

We have

$$
\begin{aligned}
\varepsilon\left(g 1_{(1)}\right) \varepsilon\left(1_{(2)} h\right) & =\varepsilon\left(g \overline{e^{(1)}}\right) \varepsilon\left(e^{(2)} h\right)=\phi\left(C\left(g \overline{e^{(1)}}\right)(1)\right) \phi\left(C\left(e^{(2)} h\right)(1)\right) \\
& =\phi\left(C(g)\left(e^{(1)}\right)\right) \phi\left(e^{(2)} C(h)(1)\right) \\
& =\phi(C(g)(C(h)(1)))=\phi(C(g h)(1))=\varepsilon(g h)
\end{aligned}
$$

and

$$
\begin{aligned}
\varepsilon\left(g 1_{(2)}\right) \varepsilon\left(1_{(1)} h\right) & =\phi\left(C\left(g e^{(2)}\right)(1)\right) \phi\left(C\left(\overline{e^{(1)}} h\right)(1)\right)=\phi\left(C(g)\left(e^{(2)}\right)\right) \phi\left(C(h)(1) e^{(1)}\right) \\
& =\phi(C(g)(C(h)(1)))=\varepsilon(g h)
\end{aligned}
$$

for $g, h \in H$,

$$
\begin{aligned}
(H \otimes \Delta) \Delta(1) & =\overline{e^{(1)}} \otimes \Delta\left(e^{(2)}\right)=\overline{e^{(1)}} \otimes \overline{\tilde{e}(1)}\left(e^{(2)}\right)_{[1]} \otimes \tilde{e}^{(2)}\left(e^{(2)}\right)_{[2]} \\
& =\overline{e^{(1)}} \otimes \overline{\tilde{e}^{(1)}} e^{(2)} \otimes \tilde{e}^{(2)}=(\Delta(1) \otimes 1)(1 \otimes \Delta(1)) \\
& =\overline{e^{(1)}} \otimes e^{(2)} \overline{\tilde{e}^{(1)}} \otimes \tilde{e}^{(2)}=(1 \otimes \Delta(1))(\Delta(1) \otimes 1) .
\end{aligned}
$$

REMARK 5.6. Let $(H, \Gamma, C)$ be a $\times_{R}$-bialgebra. Then for any idempotent Frobenius system $(\phi, e)$ we obtain a weak bialgebra structure $\left(H, \Delta_{\phi}, e_{\phi}\right)$ from Theorem 5.5.

On the other hand, if a weak bialgebra structure $(H, \Delta, \varepsilon)$ is given, we obtain a $\times_{R}$-bialgebra structure from Theorem 5.5, along with an idempotent Frobenius system for the target counital subalgebra $R:=H_{t}$ from Proposition 4.2.

Assume we start with an idempotent Frobenius system on $R$ and a $\times_{R}$-bialgebra $(H, \Gamma, C)$. Consider the weak bialgebra $(H, \Delta, \varepsilon)$ obtained from it. Assuming that the maps from $R$ and from $\bar{R}$ to $H$ making $H$ an $R^{e}$-ring are injective, it is easy to see that 
$H_{t} \cong R$, and that the idempotent Frobenius system on $H_{t}$ obtained from Proposition 4.2 is the same as the idempotent Frobenius system on $R$ originally given.

On the other hand, assume we start with a weak bialgebra $(H, \Delta, \varepsilon)$, and consider the separable algebra $R=H_{t}$ with idempotent Frobenius system $(\phi, e)$ as in Proposition 4.2, and the $\times_{R}$-bialgebra $(H, \Gamma, \varepsilon)$ as in Theorem 5.1. Then for any choice of an idempotent Frobenius system $(\psi, f)$ on $R$ we obtain a weak bialgebra structure $\left(H, \Delta_{\psi}, \varepsilon_{\psi}\right)$ from Theorem 5.5. It is quite obvious that $\Delta_{\phi}=\Delta$ and $\varepsilon_{\phi}=\varepsilon$, that is, we get the original weak bialgebra back provided we choose the idempotent Frobenius system it defines. What happens if we choose another one? Then there is an invertible $t \in R$ with $e^{(1)} t^{-1} e^{(2)}=1$, $\psi(x)=\phi(t x)$ for all $x \in R$, and $f=\left(1 \otimes t^{-1}\right) e$, and we obtain

$$
\Delta_{\psi}(h)=\overline{f^{(1)}} h_{(1)} \otimes f^{(2)} h_{(2)}=h_{(1)} \otimes t^{-1} h_{(2)}
$$

and

$$
\varepsilon_{\psi}(h)=\varepsilon(t C(h)(1))=\varepsilon\left(t \varepsilon_{t}(h)\right)=\varepsilon(t h) .
$$

This kind of twisting of a weak bialgebra structure by an invertible element in the target counital subalgebra is considered by Nikshych [12]. We see that Theorem 5.1 and Theorem 5.5 relate Takeuchi's $\times_{R}$-bialgebras to weak bialgebras up to such twists, which corresponds well to the viewpoint in [12] that twistings by invertible elements in the counital subalgebra should be considered as particularly irrelevant for the structure of $H$. Weak bialgebras that are such twists of each other can simply be obtained as different weak bialgebra versions of the same $\times_{R}$-bialgebra.

6. Weak Hopf algebras are $\times_{R}$-Hopf algebras. Etingof and Nikshych have shown that a weak Hopf algebra is a Hopf algebroid in the sense of Lu.

In this section we compare the weak Hopf algebra axioms to a different notion of "Hopf algebroid", namely that of a $\times_{R}$-Hopf algebra introduced in [18]. By definition [18, Def. 3.5], a $\times_{R}$-bialgebra is a $\times_{R}$-Hopf algebra if and only if the canonical map

$$
H \otimes \frac{\otimes}{R} H \ni g \otimes h \mapsto g_{[1]} \otimes g_{[2]} h \in H \diamond H
$$

is a bijection. This is analogous to a well-known characterization of ordinary bialgebras. Moreover, the definition is backed in [18] by a characterization of $\times_{R}$-Hopf algebras through a canonical property of their module categories.

By definition, a weak bialgebra $H$ is a weak Hopf algebra if there is an endomorphism $S$ of the $k$-space $H$ such that for all $h \in H$

$$
\begin{gathered}
S\left(h_{(1)}\right) h_{(2)}=\varepsilon_{s}(h), \\
h_{(1)} S\left(h_{(2)}\right)=\varepsilon_{t}(h), \\
S\left(h_{(1)}\right) h_{(2)} S\left(h_{(3)}\right)=S(h) .
\end{gathered}
$$

The axioms imply immediately that

$$
S\left(h_{(1)}\right) \varepsilon_{t}\left(h_{(2)}\right)=S(h)=\varepsilon_{s}\left(h_{(1)}\right) S\left(h_{(2)}\right) .
$$

Hence we have, for $x \in H_{s}$,

$$
\varepsilon_{s}\left(x h_{(1)}\right) S\left(h_{(2)}\right)=S\left(h_{(1)}\right) x h_{(2)} S\left(h_{(3)}\right)=S\left(h_{(1)}\right) x \varepsilon_{t}\left(h_{(2)}\right)=S\left(h_{(1)}\right) \varepsilon_{t}\left(h_{(2)}\right) x=S(h) x .
$$


The antipode is an algebra antihomomorphism by

$$
\begin{aligned}
S(g h) & =S\left(g_{(1)} h_{(1)}\right) \varepsilon_{t}\left(g_{(2)} h_{(2)}\right)=S\left(g_{(1)} h_{(1)}\right) \varepsilon_{t}\left(g_{(2)} \varepsilon_{t}\left(h_{(2)}\right)\right) \\
& =S\left(g_{(1)} h_{(1)}\right) g_{(2)} \varepsilon_{t}\left(h_{(2)}\right) S\left(g_{(3)}\right)=S\left(g_{(1)} h_{(1)}\right) g_{(2)} h_{(2)} S\left(h_{(3)}\right) S\left(g_{(3)}\right) \\
& =\varepsilon_{s}\left(g_{(1)} h_{(1)}\right) S\left(h_{(2)}\right) S\left(g_{(2)}\right)=\varepsilon_{s}\left(\varepsilon_{s}\left(g_{(1)}\right) h_{(1)}\right) S\left(h_{(2)}\right) S\left(g_{(2)}\right) \\
& =S(h) \varepsilon_{s}\left(g_{(1)}\right) S\left(g_{(2)}\right)=S(h) S(g)
\end{aligned}
$$

and

$$
S(1)=S\left(1_{(1)}\right) 1_{(2)} S\left(1_{(3)}\right)=S\left(1_{(1)}\right) 1_{(2)} 1^{\prime}(1) S\left(1^{\prime}(2)\right)=\varepsilon_{s}(1) \varepsilon_{t}\left(1^{\prime}\right)=1 .
$$

TheOrem 6.1. Let $H$ be a weak bialgebra. Then $H$ is a weak Hopf algebra if and only if the map

$$
\beta_{0}: H \otimes H \ni g \otimes h \mapsto g_{(1)} \otimes g_{(2)} h \in H \otimes H
$$

induces an isomorphism

$$
\beta: H \underset{H_{s}}{\otimes} H \rightarrow \Delta(1)(H \otimes H) .
$$

Proof. First, assume that $H$ has an antipode $S$. Define $\bar{\beta}_{0}: H \otimes H \rightarrow H \otimes_{H_{s}} H$ by $\bar{\beta}(g \otimes h)=g_{(1)} \otimes S\left(g_{(2)}\right) h$. Then

$$
\begin{aligned}
\beta \bar{\beta}_{0}(g \otimes h) & =\beta\left(g_{(1)} \otimes S\left(g_{(2)}\right) h\right)=g_{(1)} \otimes g_{(2)} S\left(g_{(3)}\right) h=g_{(1)} \otimes \varepsilon_{t}\left(g_{(2)}\right) h \\
& =1_{(1)} g_{(1)} \otimes \varepsilon_{t}\left(1_{(2)} g_{(2)}\right) h=1_{(1)} g_{(1)} \otimes \varepsilon\left(1^{\prime}{ }_{(1)} 1_{(2)} g_{(2)}\right) 1^{\prime}{ }_{(2)} h \\
& =1_{(1)} g_{(1)} \otimes \varepsilon\left(1_{(2)} g_{(2)}\right) 1_{(3)} h=1_{(1)} g \otimes 1_{(2)} h
\end{aligned}
$$

and

$$
\bar{\beta}_{0} \beta_{0}(g \otimes h)=g_{(1)} \otimes S\left(g_{(2)}\right) g_{(3)} h=g_{(1)} \otimes \varepsilon_{s}\left(g_{(2)}\right) h=g_{(1)} \varepsilon_{s}\left(g_{(2)}\right) \otimes h=g \otimes h
$$

Thus the restriction of $\bar{\beta}_{0}$ is an inverse to $\beta$.

Now assume that $\beta$ has an inverse $\beta^{-1}$. Define $\pi: H \otimes_{H_{s}} H \rightarrow H$ by $\pi(g \otimes h)=\varepsilon_{s}(g) h$, and define $S: H \rightarrow H$ by $S(h)=\pi \beta^{-1}\left(1_{(1)} h \otimes 1_{(2)}\right)$ for $h \in H$. We claim that $S$ is an antipode for $H$. For this we first compute

$$
\begin{aligned}
S\left(h_{(1)}\right) h_{(2)} & =\pi\left(\beta^{-1}\left(1_{(1)} h_{(1)} \otimes 1_{(2)}\right)\right) h_{(2)}=\pi\left(\beta^{-1}\left(1_{(1)} h_{(1)} \otimes 1_{(2)}\right)\left(1 \otimes h_{(2)}\right)\right) \\
& =\pi\left(\beta^{-1}\left(1_{(1)} h_{(1)} \otimes 1_{(2)} h_{(2)}\right)\right)=\pi \beta^{-1}\left(h_{(1)} \otimes h_{(2)}\right)=\pi(h \otimes 1)=\varepsilon_{s}(h) .
\end{aligned}
$$

Next, we claim that the inverse of $\beta$ is the restriction of the map

$$
\gamma: H \otimes H \ni g \otimes h \mapsto g_{(1)} \otimes S\left(g_{(2)}\right) h \in \underset{H_{s}}{\otimes} H .
$$

This is verified by the calculation

$$
\begin{aligned}
\gamma \beta_{0}(g \otimes h) & =\gamma\left(g_{(1)} \otimes g_{(2)} h\right)=g_{(1)} \otimes S\left(g_{(2)}\right) g_{(3)} h=g_{(1)} \otimes \varepsilon_{s}\left(g_{(2)}\right) h \\
& =g_{(1)} \varepsilon_{s}\left(g_{(2)}\right) \otimes h=g \otimes h .
\end{aligned}
$$

Using, for $y \in H_{s}$,

$$
\begin{aligned}
S(y h) & =\pi \beta^{-1}\left(1_{(1)} y h \otimes 1_{(2)}\right)=\pi \beta^{-1}\left(1_{(1)} h \otimes 1_{(2)} \varepsilon_{t}(y)\right) \\
& =\pi \beta^{-1}\left(1_{(1)} h \otimes 1_{(2)}\right) \varepsilon_{t}(y)=S(h) \varepsilon_{t}(y),
\end{aligned}
$$

we find

$$
\begin{aligned}
1_{(1)} h \otimes 1_{(2)} & =\beta \beta^{-1}\left(1_{(1)} h \otimes 1_{(2)}\right)=\beta\left(h_{(1)} \otimes S\left(1_{(1)} h_{(2)}\right) 1_{(2)}\right) \\
& =\beta\left(h_{(1)} \otimes S\left(h_{(2)}\right) \varepsilon_{t}\left(1_{(1)}\right) 1_{(2)}\right)=\beta\left(h_{(1)} \otimes S\left(h_{(2)}\right)\right)=h_{(1)} \otimes h_{(2)} S\left(h_{(3)}\right) .
\end{aligned}
$$


and can apply $\varepsilon \otimes H$ to the result to obtain $\varepsilon_{t}(h)=h_{(1)} S\left(h_{(2)}\right)$. We finish the proof by calculating

$$
S\left(h_{(1)}\right) h_{(2)} S\left(h_{(3)}\right)=S\left(h_{(1)}\right) \varepsilon_{t}\left(h_{(2)}\right)=S\left(h_{(1)}\right) \varepsilon_{t} \varepsilon_{s}^{\prime}\left(h_{(2)}\right)=S\left(\varepsilon_{s}^{\prime}\left(h_{(2)}\right) h_{(1)}\right)=S(h)
$$

for all $h \in H$.

Corollary 6.2. Let $H$ be a weak bialgebra. Then the following are equivalent:

(1) $H$ is a weak Hopf algebra.

(2) The associated $\times_{R}$-bialgebra $H$ is a $\times_{R}$-Hopf algebra.

Proof. The identity induces an isomorphism $\gamma \Delta(1)(H \otimes H) \rightarrow H \diamond H$ by Proposition 5.3. The composition $\gamma \beta$ is the map

$$
H \underset{H_{s}}{\otimes} H \ni g \otimes h \mapsto g_{[1]} \otimes g_{[2]} h \in H \diamond H
$$

required to be bijective in the definition of a $\times_{R}$-Hopf algebra.

For ordinary Hopf algebras, a well-known application of the characterization Theorem 6.1 is due to Nichols [11]: Any finite-dimensional quotient bialgebra $H / I$ of a Hopf algebra $H$ is itself a Hopf algebra. Dually, every finite-dimensional subbialgebra of a Hopf algebra is itself a Hopf algebra. Our results will not be quite as striking. We cannot prove that a finite-dimensional weak subbialgebra $B \subset H$ of a weak Hopf algebra $H$ is necessarily a weak Hopf algebra. But at least we can give a criterion purely in terms of the module structure of $B$ over the source and target counital subalgebras.

To prepare, we note an observation of Nikshych and Vainerman [13, 2.1.12]:

Lemma 6.3. Let $f: B \rightarrow H$ be a homomorphism of weak bialgebras. Then $f$ induces isomorphisms $B_{t} \cong H_{t}$ and $B_{s} \cong H_{s}$

Proof. We only treat the target counital subalgebra. It is trivial to check that $f\left(B_{t}\right) \subset$ $H_{t}$. We denote the induced map $B_{t} \rightarrow H_{t}$ by $f$ again. Define

$$
g: H_{t} \ni x \mapsto \varepsilon\left(x f\left(1_{(1)}\right)\right) 1_{(2)} \in B_{t} .
$$

Then

$$
g f(x)=\varepsilon\left(f(x) f\left(1_{(1)}\right)\right) 1_{(2)}=\varepsilon\left(f\left(x 1_{(1)}\right)\right) 1_{(2)}=\varepsilon\left(x 1_{(1)}\right) 1_{(2)}=x
$$

for all $x \in B_{t}$, and

$$
f g(x)=\varepsilon\left(x f\left(1_{(1)}\right)\right) f\left(1_{(2)}\right)=\varepsilon\left(x f(1)_{(1)}\right) f(1)_{(2)}=\varepsilon\left(x 1_{(1)}\right) 1_{(2)}=x
$$

for all $x \in H_{t}$, so that $g$ is inverse to $f$.

TheOREM 6.4. Let $H$ be a weak Hopf algebra, and $B \subset H$ a finite-dimensional weak subbialgebra (i.e. subalgebra and subcoalgebra) of $H$. The following are equivalent:

(1) $B$ is a weak Hopf algebra.

(2) The right $B_{s}$-module $B$ is isomorphic to the $B_{s}$-module $B$ obtained by restricting the left $B_{t}$-module $B$ along $\varepsilon_{t}$.

Proof. As a special case of the preceding Lemma we have $B_{t}=H_{t}$ and $B_{s}=H_{s}$. For the implication $(1) \Rightarrow(2)$ we need to use that the antipode of a finite-dimensional 
quasi-Hopf algebra is bijective $[1,2.10]$. Now $S$ is an algebra antiautomorphism, and for $y \in H_{s}$

$$
S(y)=S\left(y_{(1)}\right) \varepsilon_{t}\left(y_{(2)}\right)=S\left(1_{(1)}\right) \varepsilon_{t}\left(y 1_{(2)}\right)=S\left(1_{(1)}\right) 1_{(2)} \varepsilon_{t}(y)=\varepsilon_{t}(y),
$$

so that (2) follows.

Now assume (2), and fix an isomorphism $f: B \rightarrow B$ satisfying $f\left(b \varepsilon_{s}^{\prime}(x)\right)=x f(b)$ for all $b \in B$ and $x \in H_{t}$. Then

$$
B \underset{H_{s}}{\otimes} B \ni b \otimes c \mapsto c \otimes f(b) \in B \diamond B
$$

is an isomorphism of vector spaces, hence its domain and codomain have the same dimension. The canonical map

$$
B \underset{H_{s}}{\otimes} B \ni b \otimes c \mapsto b_{[1]} \otimes b_{[2]} c \in B \diamond B
$$

is the restriction (note all the modules that occur are projective) of the canonical map for $H$, hence injective, hence bijective, so that $B$ is a quasi-Hopf algebra.

With essentially the same proof we can show:

TheOREM 6.5. Let $H$ be a weak Hopf algebra, and $B=H / I$ a finite-dimensional quotient weak bialgebra (i.e. I is a coideal and an ideal). The following are equivalent:

(1) $B$ is a weak Hopf algebra.

(2) The right $B_{s}$-module $B$ is isomorphic to the $B_{s}$-module $B$ obtained by restricting the left $B_{t}$-module $B$ along $\varepsilon_{t}$.

7. Duality. In [18, Sec. 5] we have discussed a notion of skew pairing and duality suitable for $\times_{R}$-bialgebras. Let $R$ be a $k$-algebra and $H, \Lambda$ two $\times_{R}$-bialgebras. We have defined [18, Def. 5.1] a skew pairing between $\Lambda$ and $H$ to be a $k$-linear map $\tau: \Lambda \otimes H \rightarrow R$ satisfying

$$
\begin{array}{rlrl}
\tau((r \otimes \bar{s}) \xi(t \otimes \bar{u}) \mid h) v & =r \tau(\xi \mid(t \otimes \bar{v}) h(u \otimes \bar{s})) \\
\tau(\xi \mid g h) & =\tau\left(\overline{\tau\left(\xi_{[2]} \mid h\right)} \xi_{[1]} \mid g\right), & & \tau(\xi \mid 1)=C(\xi)(1), \\
\tau(\xi \zeta \mid g) & =\tau\left(\xi \mid \tau\left(\zeta \mid g_{[1]}\right) g_{[2]}\right), & & \tau(1 \mid h)=C(h)(1)
\end{array}
$$

for all $r, s, t, u, v \in R, \xi, \zeta \in \Lambda$ and $g, h \in L$.

As pointed out in [18], it is essential that we define a skew pairing rather than a pairing in this situation. (An alternative chosen by Kadison and Szlachányi [9] is to consider pairings between "left" and "right" bialgebroids.)

For weak bialgebras it is no problem to define a Hopf algebra pairing, of course, though the problem for $\times_{R}$-bialgebras has its counterpart in the fact that the source counital subalgebra of the dual of a finite-dimensional weak bialgebra $H$ is canonically isomorphic to the target rather than the source counital subalgebra of $H$.

We define a skew pairing between weak bialgebras $\Lambda, H$ to be a linear map $\tau_{0}: \Lambda \otimes H \rightarrow$ $k$ satisfying

$$
\begin{array}{ll}
\tau_{0}(\xi \mid g h)=\tau_{0}\left(\xi_{(1)} \mid g\right) \tau_{0}\left(\xi_{(2)} \mid h\right), & \tau_{0}(\xi \mid 1)=\varepsilon(\xi), \\
\tau_{0}(\xi \zeta \mid h)=\tau_{0}\left(\zeta \mid h_{(1)}\right) \tau_{0}\left(\xi \mid h_{(2)}\right), & \tau_{0}(1 \mid h)=\varepsilon(h)
\end{array}
$$

for $\xi, \zeta \in \Lambda$ and $g, h \in H$. 
For the rest of the section let $R$ be a separable algebra with idempotent Frobenius system $(\phi, e)$.

LEMma 7.1. Let $\Lambda, H$ be two $\times_{R}$-bialgebras. If $\tau: \Lambda \otimes H \rightarrow R$ is a skew pairing of $\times_{R}$-bialgebras, then $\tau_{0}:=\phi \tau: \Lambda \otimes H \rightarrow k$ is a skew pairing between the corresponding weak bialgebras.

Proof. We first note that $\overline{e^{(1)}} h_{(1)} \otimes e^{(2)} h_{(2)}=h_{(1)} \otimes h_{(2)}$ holds in $H \otimes H$ for all $h \in H$ (and similar formulas for $\Lambda$ ), so that

$$
\tau\left(\zeta \mid h_{(1)}\right) h_{(2)}=\phi\left(\tau\left(\zeta \mid h_{(1)}\right) e^{(1)}\right) e^{(2)} h_{(2)}=\phi\left(\tau\left(\zeta \mid \overline{e^{(1)}} h_{(1)}\right)\right) e^{(2)} h_{(2)}=\tau_{0}\left(\zeta \mid h_{(1)}\right) h_{(2)}
$$

for all $\zeta \in \Lambda$ and $h \in H$. It follows that

$$
\left.\tau_{0}(\xi \zeta \mid h)=\phi \tau(\xi \zeta \mid h)=\phi\left(\tau\left(\xi \mid \tau\left(\zeta \mid h_{[1]}\right) h_{[2]}\right)\right)=\tau_{0}\left(\xi \mid \tau\left(\zeta \mid h_{(1)}\right) h_{(2)}\right)\right)=\tau_{0}\left(\xi \mid \tau_{0}\left(\zeta \mid h_{(1)}\right) h_{(2)}\right)
$$

for all $\xi, \zeta \in \Lambda$ and $h \in H$. Moreover

$$
\tau_{0}(1 \mid h)=\phi \tau(1 \mid h)=\phi(C(h)(1))=\varepsilon(h) .
$$

On the other hand, using that

$$
\overline{\tau\left(\xi_{(2)} \mid h\right)} \xi_{(1)}=\overline{e^{(1)}} \phi\left(e^{(2)} \tau\left(\xi_{(2)} \mid h\right)\right) \xi_{(1)}=\overline{e^{(1)}} \xi_{(1)} \phi\left(\tau\left(e^{(2)} \xi_{(2)} \mid h\right)\right)=\xi_{(1)} \tau_{0}\left(\xi_{(2)} \mid h\right)
$$

for $\xi \in \Lambda$ and $h \in H$, we find

$$
\tau_{0}(\xi \mid g h)=\phi \tau(\xi \mid g h)=\phi \tau\left(\overline{\tau\left(\xi_{[2]} \mid h\right)} \xi_{[1]} \mid g\right)=\tau_{0}\left(\overline{\tau\left(\xi_{(2)} \mid h\right)} \xi_{(1)} \mid g\right)=\tau_{0}\left(\xi_{(1)} \tau_{0}\left(\xi_{(2)} \mid h\right) \mid g\right)
$$

for all $\xi \in \Lambda, g, h \in H$, while

$$
\tau_{0}(\xi \mid 1)=\phi \tau(\xi \mid 1)=\phi(C(\xi)(1))=\varepsilon(\xi) .
$$

By (7.1), a skew pairing between $\times_{R}$-bialgebras $\Lambda$ and $H$ defines a map $\Lambda \rightarrow$ $\operatorname{Hom}_{\bar{R}-}(H, R)$. In the case that $H$ is finitely generated projective, there is a unique $\times_{R}$-bialgebra structure on $H^{\vee}:=\operatorname{Hom}_{\bar{R}-}(H, R)$ such that evaluation defines a skew pairing $H^{\vee} \otimes H \rightarrow k$, see [18, Thm. 5.13].

In our situation, where $R$ is separable, any $R$-module is projective, so $H$ is a finitely generated projective left $\bar{R}$-module if and only if $H$ is finite-dimensional over $k$. Then the vector space dual $H^{*}$ of the weak bialgebra $H$ has a natural weak bialgebra structure, consisting of the usual dual algebra of the coalgebra $H$, and the dual coalgebra of the algebra $H$. Note that $\left(H^{*}\right)^{\text {op }}$ has a skew pairing with $H$.

Proposition 7.2. Let $H$ be a finite-dimensional $\times_{R}$-bialgebra. Then the weak bialgebra corresponding to $H^{\vee}$ is the opposite $\left(H^{*}\right)^{\text {op }}$ of the dual $H^{*}$ of the weak bialgebra corresponding to $H$.

Proof. Evaluation of $H^{\vee}$ on $H$ defines a skew pairing of $\times_{R}$-bialgebras which is nondegenerate in its right argument by definition of $H^{\vee}$, and also in its left argument since $H$ is finitely generated projective as a left $\bar{R}$-module.

We have seen that $\tau$ induces a skew pairing $\tau_{0}: H^{\vee} \otimes H \rightarrow k$ of weak bialgebras. It only remains to verify that $\tau_{0}$ is nondegenerate.

So let first $\xi \in H^{\vee}$, and assume that $\tau_{0}(\xi \mid h)=0$ for all $h \in H$. Then for all $h \in H$ we have $0=\phi\left(\tau\left(\xi \mid \overline{e^{(1)}} h\right)\right) e^{(2)}=\phi\left(\tau(\xi \mid h) e^{(1)}\right) e^{(2)}=\tau(\xi \mid h)$ and hence $\xi=0$ by definition of $H^{\vee}$. 
By a parallel argument we can show that $\tau_{0}$ is nondegenerate in the left argument as well.

REMARK 7.3. Let $(H, \Delta)$ be a finite-dimensional weak bialgebra with counital subalgebra $R$. Let $(H, \Gamma)$ be the associated $\times_{R}$-bialgebra. To distinguish, let ${ }^{(H, \Delta)} \mathcal{M}$ denote the category of left comodules over the ordinary $k$-coalgebra $H$, and let ${ }^{(H, \Gamma)} \mathcal{M}$ denote the category of left comodules over the $\times_{R}$-bialgebra $H$. By [18, Cor. 5.15] one has an equivalence of monoidal categories ${ }^{H} \mathcal{M} \cong H^{\vee} \mathcal{M}$. By Proposition 5.3 and Proposition 7.2 we may replace $H^{\vee} \mathcal{M}$ by the module category $\left(H^{*}\right)^{\text {op }} \mathcal{M}$ over the opposite of the dual weak bialgebra, which in turn is the comodule category ${ }^{(H, \Delta)} \mathcal{M}$ over the weak bialgebra $H$. Combining, we have a category equivalence ${ }^{(H, \Delta)} \mathcal{M} \cong(H, \Gamma) \mathcal{M}$, which we will now derive more directly, and without using finiteness.

Proposition 7.4. Let $(H, \Delta)$ be a weak bialgebra, and $(H, \Gamma)$ the associated $\times_{R^{-}}$ bialgebra.

(1) Let $M$ be a comodule over the $\times_{R}$-bialgebra $H$, with comodule structure $\lambda: M \ni$ $m \mapsto m_{[-1]} \otimes m_{[0]} \in H \times_{R} M$. Then the underlying vector space of $M$ is a left $H$-comodule over the $k$-coalgebra $H$ with comodule structure

$$
\delta: M \ni m \mapsto \overline{e^{(1)}} m_{[0]} \otimes e^{(2)} m_{[0]} \in H \otimes M .
$$

(2) Let $M$ be a left $H$-comodule over the $k$-coalgebra $H$, with comodule structure map $\delta$. Then $M$ is an R-bimodule by

$$
r m s:=\varepsilon\left(r m_{(-1)} s\right) m_{(0)}
$$

and a left $H$-comodule for the $\times_{R}$-bialgebra $H$ with the comodule structure $\lambda$ such that

$$
M \stackrel{\lambda}{\rightarrow} H \times_{R} M \subset H \diamond M
$$

is the composition

$$
M \stackrel{\delta}{\rightarrow} H \otimes M \rightarrow H \diamond M
$$

in which the second map is the canonical epi.

The two constructions describe a bijection between the two types of comodule structures on a given $k$-vector space $M$. In particular, one has a category equivalence ${ }^{(H, \Delta)} \mathcal{M} \cong$ $(H, \Gamma) \mathcal{M}$.

Proof. If we assume that $M$ is a comodule over the $\times_{R}$-bialgebra $H$, then the calculation showing that it is a comodule over the coalgebra $H$ as claimed in (1) is a spitting image of the proof that $H$ is an ordinary coalgebra in Theorem 5.5.

So assume that $M$ is a comodule over the ordinary coalgebra $H$. The bimodule structure (7.4) was first defined by Nill [15, Prop. 4.1] under the assumption that $H$ is finitedimensional. As a first indication that the structure is appropriate, note that we have

$$
\varepsilon\left(r h_{(1)} s\right) h_{(2)}=r h s
$$

for all $r, s \in R$ and $h \in H$ by (4.6). To see that (7.4) defines a bimodule structure, we 
only make a sample calculation, say of associativity of the left module structure:

$$
\begin{aligned}
r(s m) & =\varepsilon\left(s m_{(-1)}\right) r m_{(0)}=\varepsilon\left(s m_{(-2)}\right) \varepsilon\left(r m_{(-1)}\right) m_{(0)} \\
& =\varepsilon\left(r \varepsilon\left(s m_{(-1)(1)}\right) m_{(-1)(2)}\right) m_{(0)} \stackrel{(7.5)}{=} \varepsilon\left(r\left(s m_{(-1)}\right)\right) m_{(0)}=(r s) m .
\end{aligned}
$$

The calculation for associativity of the right $R$-module structure, and compatibility of the left and right module structures, are analogous.

Once we note now (compare $[15,(4.24)]$ ) that

$$
\begin{aligned}
m_{(-1)} 1_{(1)} \otimes m_{(0)} 1_{(2)} & =m_{(-2)} 1_{(1)} \otimes \varepsilon\left(m_{(-1)} 1_{(2)}\right) m_{(0)} \\
& =\left(m_{(-1)}\right)_{(1)} \varepsilon\left(\left(m_{(-1)}\right)_{(2)}\right) \otimes m_{(0)}=m_{(-1)} \otimes m_{(0)}
\end{aligned}
$$

holds for $m \in M$, the rest of the proof of (2) is again the same as the proof that $H$ is a $\times_{R}$-bialgebra in Theorem 5.1.

We omit showing that the two constructions described in (1) and (2) are inverse to each other.

REMARK 7.5. Using (4.8) in Remark 5.2, we can describe the tensor product of two left $H$-comodules $M$ and $N$, which is their tensor product $M \otimes_{R} N$, by the isomorphic subspace

$$
\left\{\varepsilon\left(m_{(-1)} n_{(-1)}\right) m_{(0)} \otimes n_{(0)} \mid m \in M, n \in N\right\} \subset M \otimes N
$$

since we have

$$
\begin{aligned}
m e^{(1)} \otimes e^{(2)} n & =\varepsilon\left(m_{(-1)} \varepsilon_{t}\left(1_{(1)}\right)\right) \varepsilon\left(1_{(2)} n_{(-1)}\right) \otimes m_{(0)} \otimes n_{(0)} \\
& =\varepsilon\left(m_{(-1)} 1_{(1)}\right) \varepsilon\left(1_{(2)} n_{(-1)}\right) m_{(0)} \otimes n_{(0)}=\varepsilon\left(m_{(-1)} n_{(-1)}\right) m_{(0)} \otimes n_{(0)} .
\end{aligned}
$$

for $m \in M$ and $n \in N$. Both versions of a tensor product in the category of $H$-comodules (the tensor product over $R$ and the version that is a subspace of the tensor product over $k$ ) were discussed and compared by Nill [15, Sec. 4] in the case where $H$ is finite-dimensional.

\section{References}

[1] G. Böhm, F. Nill and K. Szlachányi, Weak Hopf algebras I: Integral theory and $C^{*}$ structure, J. Algebra 221 (1999), 385-438.

[2] G. Böhm and K. Szlachányi, A coassociative $C^{*}$-quantum group with nonintegral dimensions, Lett. Math. Phys. 38 (1996), 437-456.

[3] G. Böhm and K. Szlachányi, Weak Hopf algebras. II. Representation theory, dimensions, and the Markov trace, J. Algebra 233 (2000), 156-212.

[4] T. Brzeziński and G. Militaru, Bialgebroids, $\times_{A}$-bialgebras and duality, J. Algebra 251 (2002), 279-294.

[5] P. Etingof and D. Nikshych, Dynamical quantum groups at roots of 1, Duke Math. J. 108 (2001), 135-168.

[6] F. Hausser and F. Nill, Integral theory for quasi-Hopf algebras, preprint (math.QA/ 9904164).

[7] T. Hayashi, Face algebras I-A generalization of quantum group theory, J. Math. Soc. Japan 50 (1998), 293-315. 
[8] T. Hayashi, A brief introduction to face algebras, in: New Trends in Hopf Algebra Theory (La Falda, 1999), Amer. Math. Soc., Providence, RI, 2000, 161-176.

[9] L. Kadison and K. Szlachányi, Dual bialgebroids for depth two ring extensions, preprint (math.RA/0108067).

[10] J.-H. Lu, Hopf algebroids and quantum groupoids, Int. J. Math. 7 (1996), 47-70.

[11] W. D. Nichols, Quotients of Hopf algebras, Comm. Algebra 6 (1978), 1789-1800.

[12] D. Nikshych, On the structure of weak Hopf algebras, Adv. Math. 170 (2002), 257-286.

[13] D. Nikshych and L. Vainerman, Algebraic versions of a finite-dimensional quantum groupoid, in: Hopf Algebras and Quantum Groups (Brussels, 1998), Dekker, New York, 2000, 189-220.

[14] D. Nikshych and L. Vaŭnerman, Finite quantum groupoids and their applications, in: New Directions in Hopf Algebras, Math. Sci. Res. Inst. Publ. 43, Cambridge Univ. Press, Cambridge, 2002, 211-262.

[15] F. Nill, Axioms for weak bialgebras, preprint (q-alg/9708023).

[16] P. Schauenburg, Bialgebras over noncommutative rings and a structure theorem for Hopf bimodules, Appl. Categorical Structures 6 (1998), 193-222.

[17] P. Schauenburg, Face algebras are $\times_{R}$-bialgebras, in: Rings, Hopf Algebras, and Brauer Groups, S. Caenepeel and A. Verschoren (eds.), Marcel Dekker, 1998, 275-285.

[18] P. Schauenburg, Duals and doubles of quantum groupoids ( $\times_{R}$-bialgebras), in: New Trends in Hopf Algebra Theory, N. Andruskiewitsch, W. R. Ferrer Santos, and H.-J. Schneider (eds.), Contemp. Math. 267, Amer. Math. Soc., 2000, 273-299.

[19] M. E. Sweedler, Groups of simple algebras, Publ. Math. I.H.E.S. 44 (1974), 79-189.

[20] M. Takeuchi, Groups of algebras over $A \otimes \bar{A}$, J. Math. Soc. Japan 29 (1977), 459-492.

[21] P. Xu, Quantum groupoids, Comm. Math. Phys. 216 (2001), 539-581. 